\title{
Invasive colon cancer, but not non-invasive adenomas induce a gradient effect of Wnt pathway receptor frizzled 1 (Fz1) expression in the tumor microenvironment
}

\author{
Kestutis Planutis ${ }^{1}$, Marina Planutiene ${ }^{1}$, Anthony V Nguyen², Mary Pat Moyer ${ }^{3}$ and Randall F Holcombe ${ }^{1 *}$
}

\begin{abstract}
Background: Wht signaling in the colon cancer tumor microenvironment (TME) may affect cancer biologic properties including invasion and metastatic dissemination. Prior reports have suggested that the expression of select frizzled (Fz) receptors may be altered in cancers and in the TME.

Methods: Colon cancer, colonic adenoma and normal colonic mucosal specimens were obtained under institutional review board approval and analyzed for the expression of Fz1 and Fz2 by confocal fluorescent immunohistochemistry and Wnt-specific membrane array. In vitro, the effect of Wnt3a on Fz1 expression was examined in normal-derived NCM460 cells by qRT-PCR and immunohistochemistry.

Results: Fz1 was expressed in colon cancer and villous adenomas but not in more benign tubular adenomas. Fz1 expression was seen in normal colonic mucosa in close proximity to colon cancer, but not villous or tubular adenomas. Normal colonic mucosa distant from colon cancer did not express Fz1. Fz2 was expressed ubiquitously in cancer, adenomas and normal colonic mucosa. Fz1 expression was induced by Wnt3a in a normal colon mucosa-derived cell line in vitro.

Conclusions: Fz1 is a Wht responsive gene in colon-derived tissues. Fz1 expression exhibited increased expression in normal mucosa only in close proximity to colon cancer. This field effect was not seen with pre-malignant adenomas and may be due to Wnt/ $\beta$-catenin signaling within the TME. Fz1 may represent a new TME-directed therapeutic target for patients with colon cancer.
\end{abstract}

Keywords: Wnt signaling, Colon cancer, Tumor microenvironment, Fz receptors, Wht3a

\section{Background}

There is increasing recognition that tumor biology is modulated by signals derived from, and received by cells within the tumor microenvironment (TME) [1]. Interactions with non-transformed cells in the TME may facilitate invasion and metastases, or, alternatively, inhibit tumor progression. Enhanced Wnt pathway signaling has been recognized as a hallmark of colon cancer with over $85 \%$ of these malignancies harboring mutations within the pathway that lead to constitutive activation [2]. However, the strength of the Wnt

\footnotetext{
* Correspondence: randall.holcombe@mssm.edu

${ }^{1}$ Division of Hematology/Oncology, Tisch Cancer Institute, Mount Sinai School of Medicine, New York, NY, USA

Full list of author information is available at the end of the article
}

signal is modulated by factors within the TME, including Wnt ligands which augment canonical Wnt signaling and soluble inhibitors which suppress it $[3,4]$.

Wnt activity in colon cancer stem cells is modulated by myofibroblast-secreted factors in the TME [5] and other stromal cells may similarly regulate Wnt activity in colon cancer in a paracrine fashion [6]. Interactions of malignant with stromal cells may affect Wnt signaling directly [7] or via autocrine feedback loops [8] from tumor cells overexpressing secreted Wnt ligands [9]. The invasion margin of colon cancer is characterized by nuclear b-catinin suggesting increased Wnt pathway activation at the cancer: stroma interface [10]. While most studies have focused on the effects of stroma on the biologic behavior of the cancer 
cells, Wnt signaling in the TME can also directly affect the tumor stroma, with subsequent secondary effects on cancer invasion and progression [11].

Frizzled receptors are 7-transmembrane proteins which serve, in conjunction with LRP5/6 co-receptors as the binding partners for extracellular Wnt ligands to initiate the Wnt signal. Conceptually, the signals transduced progress along one of two intracellular pathways - canonical, resulting ultimately in the accumulation of $\beta$-catenin in the nucleus where it promotes transcriptional activation in conjunction with the LEF/TCF family of DNA binding proteins and non-canonical, resulting in activation of protein kinase $\mathrm{C}$, c-Jun-N-terminal kinases or calcium/calmodulin-dependent kinases. However, Wnt signaling is significantly more complex as there is crosstalk between canonical and non-canonical pathways, and ligands and receptors may result in differential signaling in a contextdependent fashion [12].

Few studies have examined the role of distinct frizzled receptors in the TME. Factors in the TME have been implicated in the regulation of Fz7 on colorectal cancer cells [13] and Fz7 downregulation has been linked to reduced survival, invasion and metastatic potential [14], suggesting that Fz7 may represent a therapeutic target. In this report, we describe the differential expression of Fz1 in the colon cancer TME and provide evidence of autocrine regulation in tumor cells and paracrine regulation by factors within the TME on Fz1 expression in non-malignant mucosal epithelium, as well as lack of paracrine regulation in proximity to pre-malignant colon adenomas. To our knowledge, this is the first report of a field effect of a specific frizzled receptor in the colon tumor microenvironment.

\section{Materials and methods}

\section{Tissue acquisition}

Over 20 distinct de-identified colon cancer and corresponding normal colonic mucosa was obtained from archived specimens at UCIrvine under a non-human subjects exemption by the UCIrvine institutional review board. Adenoma specimens with biopsies of corresponding normal mucosa were obtained from 12 different patients following informed consent under a UCIrvine institutional review board approved protocol from patients undergoing elective colonoscopic screening as described previously [15]. All research was performed in compliance with the Declaration of Helsinki.

\section{Cell culture}

The NCM460 cell line was purchased from INCELL Corporation (San Antonio, TX, USA) and cultured in M3 media supplemented with $10 \% \mathrm{FBS}, 100 \mathrm{U} / \mathrm{ml}$ penicillin and $100 \mathrm{mg} / \mathrm{ml}$ streptomycin at $37^{\circ} \mathrm{C}$ in a $95 \%$ humidified atmosphere with $5 \% \mathrm{CO} 2$. This epithelial cell line was derived from normal colonic mucosa and is not tumor igenic though, over time in culture it has acquired some transformation-associated characteristics [16]. NCM460 is not transfected with any exogenous genetic information.

\section{WNT3a conditioned medium (CM)}

Wnt3a CM and control CM were prepared using the LWnt3A cell line (ATCC\#CRL-2647) and the parental cell line (ATCC \#CRL-2648) according to procedures perfected in the laboratory of Dr. Roel Nusse [17]; for a preparation protocol, see http://www.stanford.edu/group/ nusselab/cgi-bin/wnt/. Cells were analyzed after $24 \mathrm{~h}$ of Wnt3a treatment for Fz1 expression.

\section{Immunohistochemistry}

Immunohistochemistry for Fz1 and Fz2 were performed with specific anti-Fz1 antibodies (Genentech, Inc., \#GTX71477) and anti-Fz2 antibodies, (Genentech, Inc. \#GTX71817), $1 \mathrm{mg} / \mathrm{ml}$, at 1:200 dilution. Paraffinimbedded slides were deparaffinized and sodium citrate buffer pH $620 \mathrm{~min}$ at $99^{\circ} \mathrm{C}$ was used as an antigen retrieval agent. Santa Cruz Biotechnology rabbit ABC Staining System (SC-2018) was used according to the manufacturer's protocol, and the signal was amplified by $\mathrm{TSA}^{\mathrm{T}}$ Fluorescein Tyramide Reagent from Perkin Elmer (SAT701001EA). Slides were stained with DAPI and covered with anti-fade embedding medium (ProLong Gold antifade reagent, Invitrogen P36934). Confocal microscopy was performed with a Carl Zeiss LSM 510 and 40x fluorescent objective.

Quantitative real-time polymerase chain reaction (qRT-PCR) Fz1 expression in NCM460 was determined by qRTPCR with Fz1 specific primers. The efficiency of the Fz1 and actin primers was equivalent. Primer pairs were obtained from Qiagen (Valencia, CA) with cycling parameters as defined by the manufacturer. For qRTPCR experiments, the relative RNA expression was calculated by the comparative threshold cycle method.

\section{Wnt-specific membrane array}

Fz1 mRNA expression in adenomas and normal mucosa was defined with cDNA generated from tissue-derived RNA and analyzed with a GEArray Q Series Human Wnt Signaling Pathway Gene Array (SuperArray Bioscience, Frederick, MD). GEArray expression analysis software was utilized for background normalization, correction for different degrees of exposure, and normalization with multiple housekeeping gene controls on each membrane.

\section{Statistical analysis}

Fz1 mRNA and protein expression in NCM460 cells was compared with an unpaired $t$-test between experimental (Wnt3a CM) and control (L-Cell CM) groups. 
Expression of Fz1 in adenomas, was analyzed across the 3 groups by 1-way ANOVA with a post-hoc Newman Kuels test. Individual subgroups were also compared by unpaired $t$-test.

\section{Results}

Expression of Fz1 and Fz2 receptors in colon cancer tumor microenvironment

Fluorescent immunohistochemistry with specific antiFz1 and anti-Fz2 antibodies was performed on tissue sections from colon cancer, adjacent normal colonic mucosa and distant normal colonic mucosa. All experiments included secondary antibody only controls on sequential tissue sections. Fz1 and Fz2 were both expressed in colon cancer tissues. Fz1 was detected in histologically normal mucosa in close proximity to the neoplastic cells (see Figure 1, panels $\mathrm{H}$ and P). The normal mucosa examined was adjacent to an invasive margin of the tumor. More distant from the tumor, however, Fz1 expression was not seen in normal mucosa demonstrating a field effect in the tumor microenvironment with respect to expression of this specific Wnt receptor. In contrast,

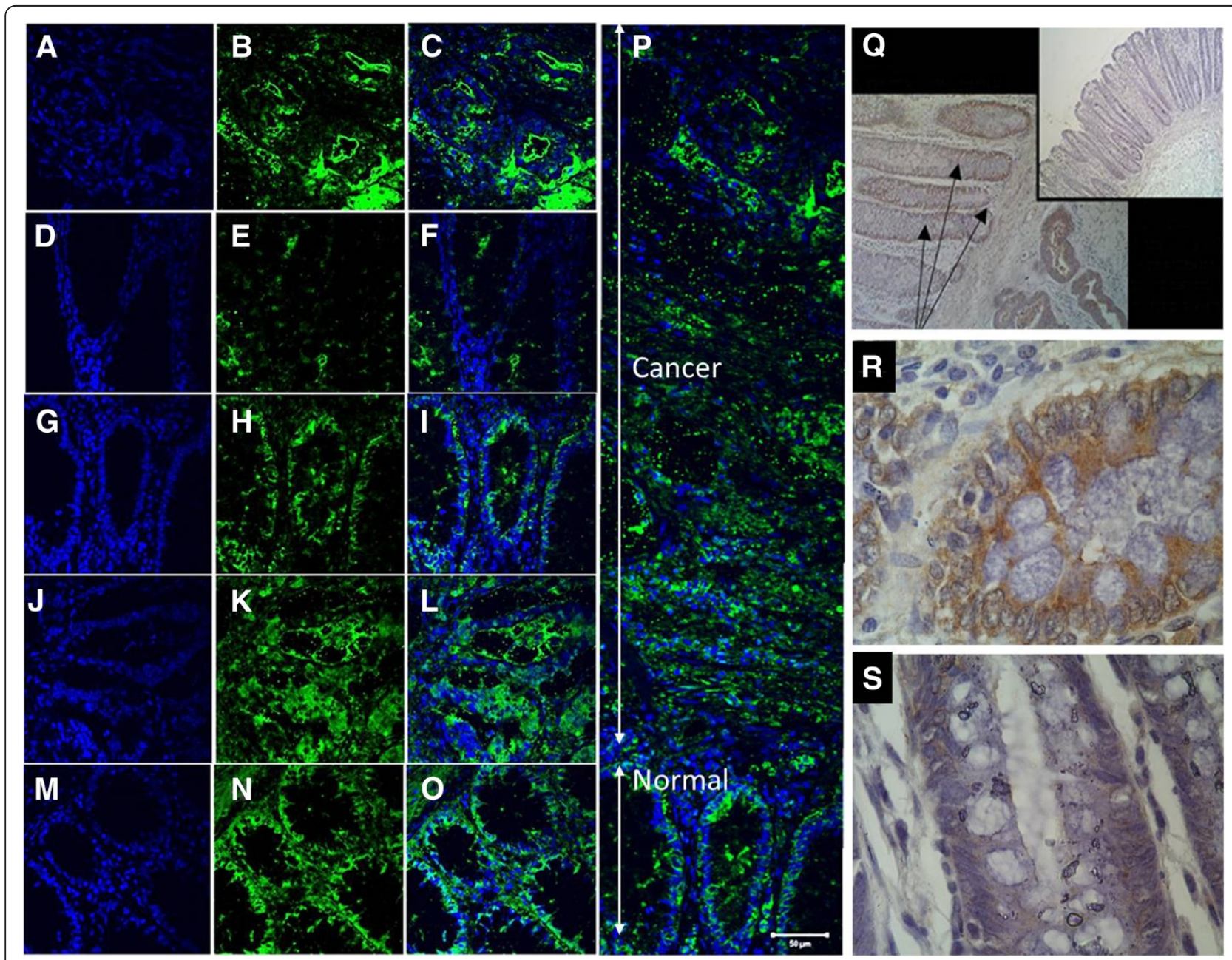

Figure 1 Expression of frizzled receptors by fluorescent immunohistochemistry in patient derived samples. Panels A, B, C: Fz1 expression in colon cancer - DAPI (A), anti-Fz1 antibody (B), merged images $(\mathbf{C})$ - showing uniform expression. Panels D, E, F: Fzl expression in normal colonic mucosa distant $(>2 \mathrm{~cm})$ from colon cancer - DAPI (D), anti-Fzl antibody $(\mathbf{E})$, merged images $(\mathbf{F})$ - showing lack of significant expression. Panels G, H, I: Fz1 expression in normal colonic mucosa adjacent to colon cancer - DAPI (G), anti-Fz1 antibody (H), merged images (I) - showing significant Fz1 expression. Panels $\mathbf{J}, \mathbf{K}, \mathbf{L}$ : Fz2 expression in colon cancer - DAPI (J), anti-Fz2 antibody (K), merged images (L) - showing uniform expression. Panels $\mathbf{M}, \mathbf{N}, \mathbf{O}$ : Fz2 expression in normal colonic mucosa distant (>2 cm) from colon cancer - DAPI (M), anti-Fz2 antibody $(\mathbf{N})$, merged images ( $\mathbf{O})$ - showing uniform expression. Panel P: Composite of multiple merged images of Fz1 expression in colon cancer and normal colonic mucosa adjacent to tumor showing expression in both the cancer and in the adjacent normal mucosa. Scale bar $=50$ um. Panel Q: DAB immunohistochemistry with anti-Fz1/(2) antibody of colon cancer, normal colonic mucosa adjacent to the tumor (arrows) and normal colonic mucosa distant from the tumor (insert) showing significant expression in the cancer and in the adjacent normal colonic mucosa. Panel R: Higher magnification from image displayed in Panel $\mathbf{Q}$ of normal colonic mucosa adjacent to colon cancer showing expression. Panel $\mathbf{S}$ : Higher magnification from image displayed in Panel $\mathbf{Q}$ of normal colonic mucosa distant from colon cancer showing lack of expression. 
Fz2 was expressed ubiquitously in normal colonic mucosa in addition to its expression in colon cancer.

Induction of Fz1 expression in vitro

In tissue culture, normal colon-derived NCM460 cells were evaluated for expression of cell surface Fz1 in response to exogenous Wnt ligand Wnt3a. These experiments demonstrate that Fz1 is a Wnt-responsive gene, with increased expression in response to Wnt3a. The Wnt3a response was manifested by both an increase in Fz1 mRNA ( $<<0.005$; Figure 2, panel A) and Fz1 protein (light intensity, $\mathrm{p}<0.001$; Figure 2, panel D), suggesting that enhanced transcription is the primary mechanism leading to increased Fz1 expression in response to Wnt3a.

\section{Expression of Fz1 in colonic adenomas}

Colonic adenomas were evaluated for Fz1 expression in order to define the stage in the neoplastic progression where Fz1 expression begins and whether the field effect for Fz1 noted in normal mucosa adjacent to colon cancer is specific for invasive carcinoma. Using a Wnt specific membrane array, Fz1 mRNA could be detected at low levels in both normal colonic mucosa and tubular adenomas. However, Fz1 mRNA was markedly increased in villous adenomas in comparison to normal mucosa and tubular adenomas $(\mathrm{p}=0.01761$ way ANOVA, $\mathrm{p}<0.05$ Newman-Kuels for both comparisons; $\mathrm{p}=0.024$ villous vs. tubular by unpaired $t$-test; $\mathrm{p}=0.012$ villous vs. normal by unpaired $t$-test). Villous adenomas have a substantially higher malignant potential than tubular adenomas.

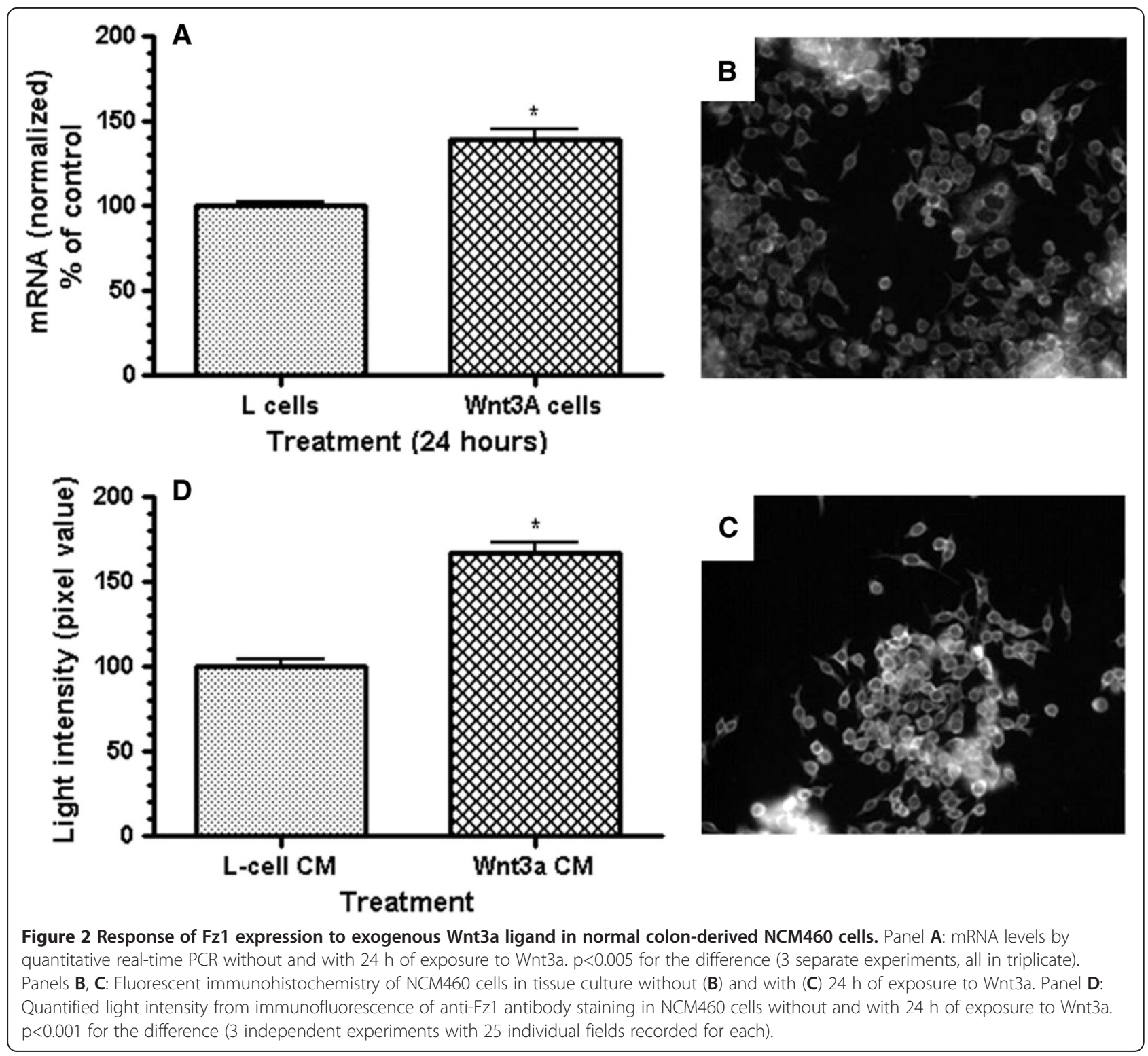


At the protein level, villous adenomas had abundant Fz1 expression (Figure 3, panels $\mathrm{G}$ and $\mathrm{H}$ ) but no expression was detected in normal mucosa or tubular adenomas, suggesting that the molecular switch or conditions within the tumor microenvironment permissive for Fz1 expression occur in the transition from tubular to villous histologies.. In contrast to what was seen with invasive colon cancer, however, no field effect of the expression of Fz1 was seen in close proximity to non-invasive adenomas (Figure 3, panel I), including villous adenomas which themselves expressed Fz1 protein.

\section{Discussion/conclusions}

The expression of the Wnt pathway receptors Fz1 and Fz2 has been previously implicated in cancer development and progression. Their expression is reported to be increased in breast cancer in comparison to normal breast epithelium [18]. There is also prior evidence using older, less specific antibodies that Fz1 or Fz2 expression is increased at the invasion margin of poorly differentiated colon cancer [9]. Fz1 mediates Wnt/ $\beta$-catinin (canonical) signaling in neuroblastoma [19] and serves as a receptor for Wnt3a in both macrophages [20] and pheochromocytomaderived PC12 cells [21]. In both these latter systems, Wnt3a/Fz1 interactions induce signaling along the canonical Wnt pathway. However, there is one report that Fz1 is antagonistic of Wnt/ $\beta$-catinin signaling in a Xenopus cap assay and secondary axis formation assay [22], suggesting that the specific activity of Fz1 may be context dependent. The role of Fz2 is less well studied though it appears to

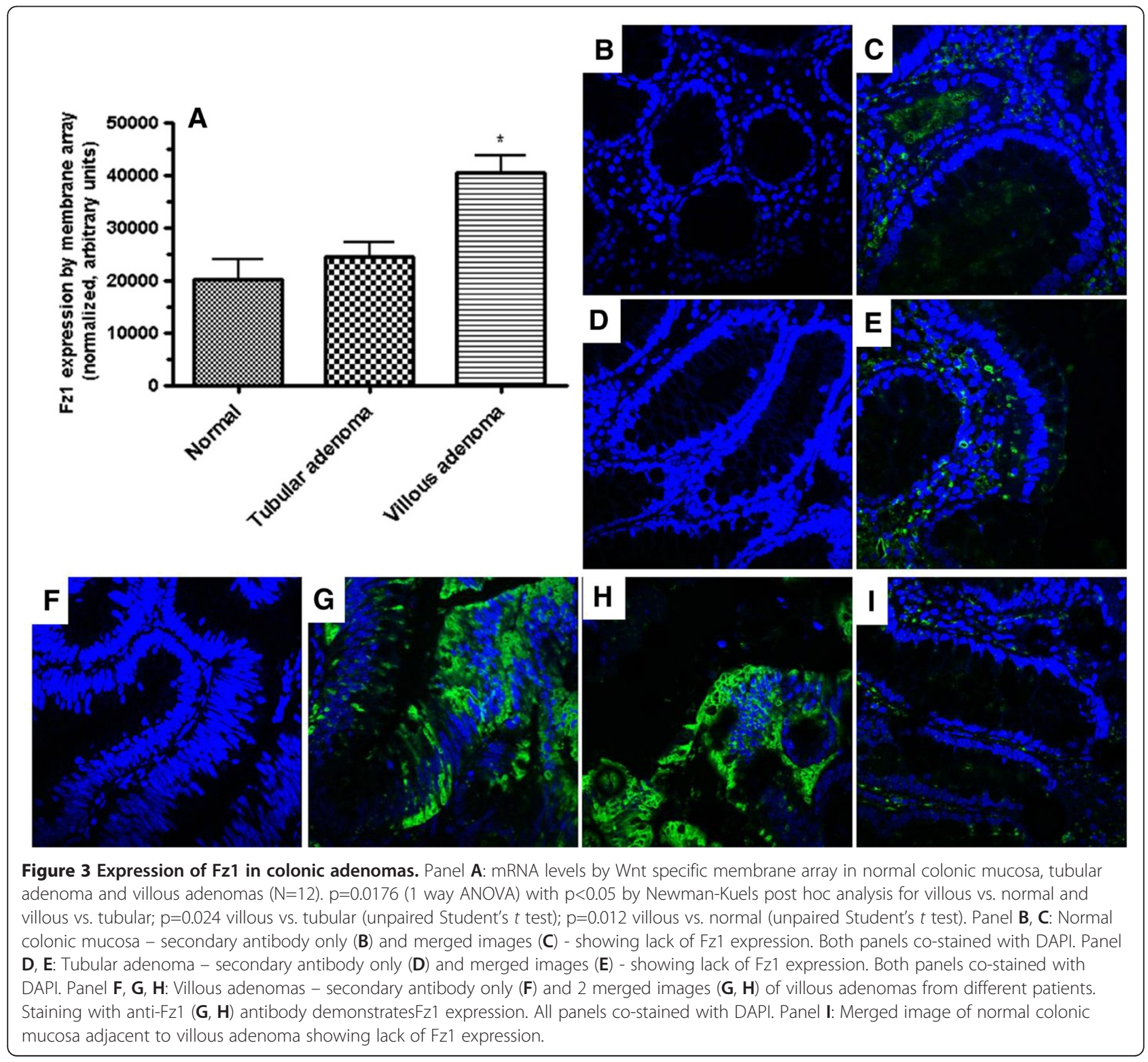


bind to both Wnt3a, which promotes Wnt/ $/$-catinin signaling and Wnt5a which inhibits it [23].

What is clear from this report is that Fz1 expression is tightly regulated in the tumor microenvironment. As both an effector molecule for Wnt signaling and also, as we have shown here, as a Wnt responsive gene, Fz1 may function as part of a positive autocrine feedback loop in colon cancer cells. Whether this effect is limited to Wnt3a which promotes Fz1 transcription and to which Fz1 also binds requires further study. In the tumor microenvironment, a field effect of Fz1 expression exists suggesting paracrine regulation of this receptor. Fz1 expression on normal colonic mucosa within the TME is induced by cancer but not by non-invasive adenomas, including villous adenomas which themselves express Fz1. We speculate that this is related to the degree of Wnt, and particularly Wnt3a ligand production by the colon cancer cells. Increased nuclear localization of $\beta$ catenin at the invasion front is a characteristic of colon cancer [24] and colon cancer is known to have increased expression of various Wnt ligands [9]. While this prior work demonstrated differences in Wnt ligand expression between tumor and normal tissues, whether a gradient of Wnt3A expression within the tumors exists, possibly increased at the invasion margin, has not been investigated. Differences in the level of Wnt activity within tumors may occur, with a high activity of the Wnt pathway is observed in tumour cells located close to stromal myofibroblasts, implicating signals from the stroma may be involved [5]. Secreted factors other than Wnt ligands may also be important. For example, Suzuki and colleagues [25] have reported that epigenetic inactivation of SFRP genes leads to augmented Wnt signaling in colon cancer and this process may not be operative in pre-malignant adenomas. Lack of nuclear translocation of $\beta$-catenin in sporadic adenomas, in contract to cancer, has been described previously [26]. Finally, expression of Fz1, as well as Fz6 and Fz7, are positively correlated with $\mathrm{Wnt} / \beta$-catenin signaling in human mesenchymal cells [27] further suggesting that the regulated expression of Fz1 in the tumor microenvironment is a function of, and contributes to, Wnt signaling in the tumor:stromal mileau. While no morphologic change in the composition of stroma was identified in our study, experiments were not designed to specifically detect this. Stromal cell recruitment at tumor sites has been described previously [28]. Further investigations will be needed to define whether Fz1 and Wnt signaling play a role in this process.

In addition to its expression in colon cancer, Fz1 expression appears to be confined to pre-malignant adenomas with villous features which represent the histologic type of adenoma with greatest risk for malignant transformation [29]. Development of rapid immunofluorescence techniques that can be performed in conjunction with advanced endoscopic imaging could potentially lead to the use of Fz1 to differentiate villous from non-villous adenomas in situ. This marker may also prove a useful adjunct to routine histologic examination of colonic adenomas.

The significance of Fz1 on normal colonic mucosa in the TME requires further study. Given the known functions of $\mathrm{Wnt} / \beta$-catinin signaling, it may promote proliferation and block differentiation of colonic mucosal cells, promoting tumor invasion by accelerating mucosal turnover. Fz1 expression in the TME may modulate colon cancer progression and dissemination and therefore may represent a new TME-directed therapeutic target for patients with colon cancer.

\section{Competing interests}

$\mathrm{KP}, \mathrm{MP}, \mathrm{AVN}$ and RFH have no conflicts of interest to disclose. MPM is owner of INCELL Corporation which markets the NCM460 cell line utilized in this study.

\section{Authors' contributions}

KP and MP conducted the majority of studies outlined in this study. AVN conducted membrane array studies related to adenomas. MPM provided the cell line for in vitro studies and reviewed the data and manuscript. RFH designed the studies and provided oversight for the project overall. All authors approved the final manuscript.

\section{Acknowledgments}

\section{Grant support}

This work was supported in part by NIH grant K24CA082450 (RFH).

\section{Author details}

${ }^{1}$ Division of Hematology/Oncology, Tisch Cancer Institute, Mount Sinai School of Medicine, New York, NY, USA. 'Division of Hematology/Oncology, University of California, Irvine, Irvine, California, USA. ${ }^{3}$ INCELL Corporation, San Antonio, Tx, USA.

Received: 1 October 2012 Accepted: 20 February 2013

Published: 26 February 2013

\section{References}

1. Witz IP: Yin-yang activities and vicious cycles in the tumor microenvironment. Cancer Res 2008, 68:9-13.

2. Miyaki M, Konishi M, Kikuchi-Yanoshita R, Enomoto M, Igari T, Tanaka K, Muraoka M, Takahashi H, Amada Y, Fukayama M, Maeda Y, Iwama T, Mishima Y, Mori T, Koike M: Characteristics of somatic mutation of the adenomatous polyposis coli gene in colorectal tumors. Cancer Res 1994, 54:3011-3020.

3. Naji R, Holcombe RF, Waterman ML: Wnt signaling and colon carcinogenesis: Beyond APC. J Carcinogenesis 2011, 10:5-13.

4. Lau W, Barker N, Clevers $\mathrm{H}$ : Wnt signaling in the normal intestine and colorectal cancer. Front Biosci 2007, 12:471-491.

5. Vermeulen L, DeSousa EMF, van der Heijden M, Cameron K, de Jong JH, Borovski R, Tuynman JB, Todaro M, Merz C, Rodermond H, Sprick MR, Kemper K, Richel DJ, Stassi G, Medema JP: Wnt activity defines colon cancer stem cells and is regulated by the microenvironment. Nat Cell Bio 2010, 12:468-476.

6. Le NH, Franken P, Fodde R: Tumour-stroma interactions in colorectal cancer: converging on beta-catenin activation and cancer stemness. $\mathrm{Br}$ J Cancer 2008, 98:1886-1893.

7. Bandapalli OR, Dihlmann S, Helwa R, Macher-Geoppinger S, Weitz J, Schirmacher $\mathrm{P}$, Brand K: Transcriptional activation of the beta-catenin gene at the invasion front of colorectal liver metastases. J Pathol 2009, 218:370-379. 
8. Bafico A, Liu G, Goldin L, Harris V, Aaronson SA: An autocrine mechanism for constitutive Wnt pathway activation in human cancer cells. Cancer Cell 2004, 6:497-506

9. Holcombe RF, Marsh JL, Waterman ML, Milovanovic T, Truong T: Expression of Wnt ligands and frizzled receptors in colonic mucosa and in colon carcinoma. Mol Pathol 2002, 55:220-226.

10. Jung A, Schrauder M, Oswald U, Knoll C, Sellberg P, Palmqvist R, Niedobitek $G$, Brabletz T, Kirchner T: The Invasion Front of Human Colorectal Adenocarcinomas Shows Co-Localization of Nuclear $\beta$-Catenin, Cyclin D1, and p16INK4A and Is a Region of Low Proliferation. Am J Pathol 2001, 159:1613-1617.

11. Macheda ML, Stacker SA: Importance of Wnt signaling in the tumor stroma microenvironment. Curr Cancer Drug Targets 2008, 8:454-465.

12. Van Amerongen $R$, Nusse R: Toward an integrated view of Wnt signaling in development. Development 2009, 36:3205-3214.

13. Vincan E, Flanagan DJ, Pouliot N, BRabletz R, Spaderna S: Variable FZD7 expression in colorectal cancers indicates regulation by the tumor microenvironment. Dev Dyn 2010, 239:311-317.

14. Ueno K, Hazama S, Mitormori S, Nishioka M, Suehiro Y, Hirata H, Oka M, Imai K, Dahiya R, Hinoda Y: Down-regulation of frizzled-7 expression decreases survival, invasion and metastatic capabilities of colon cancer cells. $\mathrm{Br} \mathrm{J}$ Cancer 2009, 101:1374-1381.

15. Nguyen AV, Albers CG, Holcombe RF: Differentiation of tubular and villous adenomas based on Wnt pathway-related gene expression profiles. Int J Mol Med 2010, 26:121-5.

16. Moyer MP, Stauffer JS, Manzano LA, Tanzer LR, Merriman RL: NCM460, A normal human colon mucosal epithelial cell Line. In Vitro Cell Dev Biol Anim 1996, 32:315-317.

17. Willert K, Brown JD, Danenberg E, Duncan AW, Weissman IL, Reya T, Yates JR, Nusse R: Wnt proteins are lipid-modified and can act as stem cell growth factors. Nature 2003, 423:448-452.

18. Milovanovic T, Planutis K, Nguyen A, Marsh L, Lin F, Hope C, Holcombe RF: Expression of Wnt genes and frizzled 1 and 2 receptors in normal breast epithelium and infiltrating breast carcinoma. Int J Oncol 2004, 25:1337-1342

19. Flahaut M, Meier R, Coulon A, Nardou KA, Niggli FK, Martinet D, Bechmann JS, Joseph JM, Muehlethaler-Mottet A, Gross N: The Wnt receptor FZD1 mediates chemoresistance in neuroblastoma through activation of the Wnt/beta-catenin pathway. Oncogene 2009, 28:2245-2256.

20. Neumann J, Schaale K, Farhat K, Endermann T, Ulmer AJ, Ehlers S, Reiling N: Frizzled 1 is a marker of inflammatory macrophages and its ligand Wnt3a is involved in reprogramming Mycobacterium tuberculosisinfected macrophages. FASEB J 2010, 24:4599-4612.

21. Chacon MA, Varela-Nallar $L$, Inestrosa NC: Frizzled-1 is involved in the neuroprotective effect of Wnt3a against Abeta oligomers. J Cell Physiol 2008, 217:215-227

22. Roman-Roman S, Shi E-L, Stiot V, Hay E, Vayssiere B, Garcia R, Baron R, Rawadi $\mathrm{G}$ : Murine frizzled-1 behaves as an antogaonist of the canonical Wnt/ß-catinin signaling. J Biol Chem 2004, 279:5725-5733.

23. Sato A, Yamamoto $H$, Sakane $H$, Koyama $H$, Kikuchi A: Wnt5a regulates distinct signaling pathways by binding to frizzled 2. EMBO J 2010 29:41-54.

24. Brabletz T, Jung A, Hermann K, Guenther K, Hohenberger W, Kirchner T: Nuclear overexpression of the oncoprotein $\beta$-catenin in colorectal cancer is localized predominantly at the invasion front. Pathol Res Pract 1998, 194:701-704.

25. Suzuki H, Watkins DN, Jair K-W, Schuebel KE, Markowitz SD, Chen WD, Pretlow TP, Yang B, Akiyama Y, van Engeland M, Toyota M, Tokino T, Hinoda Y, Imai K, Herman JG, Baylin SB: Epigenetic inactivation of SFRP genes allows constitutive WNT signaling in colorectal cancer. Nat Genet 2004, 36:417-422

26. Kobayashi M, Honma T, Matsuda Y, Suzuki Y, Narisawa R, Ajioka Y, Asakura $\mathrm{H}$ : Nuclear translocation of beta-catenin in colorectal cancer. $\mathrm{Br} J \mathrm{Cancer}$ 2000, 82:1689-1693.
27. Kolben T, Perobner I, Fernsebner K, Lechner F, Geissler C, Ruiz-Heinrich L, Capovilla $S$, Jochum $M$, Neth P: Dissecting the impact of frizzled receptors in Wnt/ $\beta$-catenin signaling of human mesenchymal stem cells. Biol Chem 2012, 393:1433-1447.

28. Wels J, Kaplan RN, Rafii S, Lyden D: Migratory neighbors and distant invaders: tumor -associated niche cells. Genes Dev 2008, 22:559-574

29. Lieberman DA, Smith FW: Screening for colon malignancy with colonoscopy. Am J Gastroenterol 1991, 86:946-951.

doi:10.1186/1479-5876-11-50

Cite this article as: Planutis et al.: Invasive colon cancer, but not noninvasive adenomas induce a gradient effect of Wnt pathway receptor frizzled 1 (Fz1) expression in the tumor microenvironment. Journal of Translational Medicine 2013 11:50.

\section{Submit your next manuscript to BioMed Central and take full advantage of:}

- Convenient online submission

- Thorough peer review

- No space constraints or color figure charges

- Immediate publication on acceptance

- Inclusion in PubMed, CAS, Scopus and Google Scholar

- Research which is freely available for redistribution

Submit your manuscript at www.biomedcentral.com/submit
C Biomed Central 